\title{
Differential chemosensitivity to antifolate drugs between RAS and BRAF melanoma cells
}

\author{
Imanol Arozarena ${ }^{1 *}$, Ibai Goicoechea ${ }^{2 \dagger}$, Oihane Erice ${ }^{3 \dagger}$, Jennnifer Ferguson', Geoffrey P Margison ${ }^{4}$ \\ and Claudia Wellbrock ${ }^{*}$
}

\begin{abstract}
Background: The importance of the genetic background of cancer cells for the individual susceptibility to cancer treatments is increasingly apparent. In melanoma, the existence of a BRAF mutation is a main predictor for successful BRAF-targeted therapy. However, despite initial successes with these therapies, patients relapse within a year and have to move on to other therapies. Moreover, patients harbouring a wild type BRAF gene (including 25\% with NRAS mutations) still require alternative treatment such as chemotherapy. Multiple genetic parameters have been associated with response to chemotherapy, but despite their high frequency in melanoma nothing is known about the impact of BRAF or NRAS mutations on the response to chemotherapeutic agents.
\end{abstract}

Methods: Using cell proliferation and DNA methylation assays, FACS analysis and quantitative-RT-PCR we have characterised the response of a panel of NRAS and BRAF mutant melanoma cell lines to various chemotherapy drugs, amongst them dacarbazine (DTIC) and temozolomide (TMZ) and DNA synthesis inhibitors.

Results: Although both, DTIC and TMZ act as alkylating agents through the same intermediate, NRAS and BRAF mutant cells responded differentially only to DTIC. Further analysis revealed that the growth-inhibitory effects mediated by DTIC were rather due to interference with nucleotide salvaging, and that NRAS mutant melanoma cells exhibit higher activity of the nucleotide synthesis enzymes IMPDH and TK1. Importantly, the enhanced ability of RAS mutant cells to use nucleotide salvaging resulted in resistance to DHFR inhibitors.

Conclusion: In summary, our data suggest that the genetic background in melanoma cells influences the response to inhibitors blocking de novo DNA synthesis, and that defining the RAS mutation status could be used to stratify patients for the use of antifolate drugs.

Keywords: Melanoma, RAS, BRAF, Alkylating agents, DNA synthesis, Salvage

\section{Background}

Cutaneous melanoma is a deadly form of skin cancer that develops from melanocytes, specialized pigmented cells that reside underneath the epidermis. $50 \%$ of melanomas harbour activating mutations in the kinase BRAF, the most common being a V600E substitution [1], and 25\% harbour mutations in the GTPase NRAS. Both oncogenes stimulate the MAP-kinase (MAPK)-pathway, which is found hyperactivated in $90 \%$ of all melanomas [2]. Whereas BRAF only activates the MAPK-pathway, NRAS activates several other

\footnotetext{
* Correspondence: Imanol.Arozarena@manchester.ac.uk;

Claudia.Wellbrock@manchester.ac.uk

${ }^{\dagger}$ Equal contributors

${ }^{1}$ Manchester Cancer Research Centre, The University of Manchester, Michael Smith Building, Oxford Road, Manchester M13 9PT, UK

Full list of author information is available at the end of the article
}

effectors including Ral-GDS or PI3-kinase, which is of special relevance for melanoma [2].

It is now accepted that genetic lesions in BRAF and NRAS have different consequences in melanoma formation and it is becoming apparent that BRAF can regulate invasion and metastasis through mechanisms different to NRAS [3].

Importantly, the genetic background of melanoma also impacts on the response to therapies targeting the MAPKpathway. While there is no efficient targeted therapy against wild type BRAF melanomas, BRAF mutant (mutBRAF) melanomas are addicted to the MAPK-pathway and small molecule inhibitors targeting either mutBRAF or MEK have shown impressive clinical responses [4-7]. Unfortunately, these responses are transient, and patient relapse 
due to acquired resistance [8]. In contrast to mutBRAF melanomas, mutNRAS tumours are largely resistant to BRAF inhibitors $[9,10]$, and moreover these drugs paradoxically stimulate the MAPK-pathway [11]. Thus, despite the initial successes with BRAF targeted therapy, relapsed patients as well as the $50 \%$ of patients harbouring a wild type BRAF (including the $25 \%$ with NRAS mutations) will still require alternative treatment such as chemotherapy and/or immunotherapy.

In melanoma some of the most commonly used chemotherapeutics are the monofunctional-alkylating agents dacarbazine (DTIC) and temozolomide (TMZ), the chloroethylating agents carmustine or the bifunctional alkylating agents like cisplatin [12]. Also anti-mitotic drugs like paclitaxel and vinblastine are used in the treatment of melanoma patients (www.cruk.org). Historically the prodrug DTIC has been the first line treament with an average overall response of $20 \%$ [12]. In patients DTIC is metabolized in the microsomes of hepatocytes into MTIC, which undergoes spontaneous transformation into a toxic DNA methylating agent [13]. More recently patients are being treated with Temozolomide (TMZ), which does not require metabolic activation, but spontaneously converts into MTIC and shows a clinical response almost identical to DTIC $[13,14]$.

Notably, compared to brain tumors, melanoma responses to alkylating agents are poor. In patients with malignant melanoma, the overall response to temozolomide is around $15 \%$ compared to 47 and $61 \%$ in glioma and astrocytoma patients $[12,15]$. Interestingly mutBRAF melanoma patients have shown responses to DTIC of up to $23 \%$ [16] but, on the other hand, recent reports state that activating mutations in BRAF have no impact on the response of stage IV melanoma patients to DTIC or TMZ [17]. However, BRAF and NRAS mutation status has never been tested retrospectively for its potential as predictive marker for DTIC responses. Resistance to these alkylating agents is thought to be due to several factors, including the altered expression of components of the apoptotic and DNA damage repair machineries and to multi-drug resistance phenotype-associated proteins such as the ABC drug transporters [18-20]. Pre-clinical studies using melanoma and glioma cells and xenografts have shown that expression of the DNA repair protein $O^{6}$-methylguanineDNA-methyl-transferase (MGMT) confers resistance to mono-alkylating agents such as DTIC, TMZ and carmustine [21]. However, this has not been successfully translated into the clinic and the use of MGMT inactivating agents to sensitise cancer cells to alkylating drugs has not provided any clinical benefit [21]. Thus, in contrast to targeted therapy, and despite extensive studies into DNA repair mechanisms in relation to tumour response, there are no good markers to predict a patient's response to chemotherapy. Since in melanoma the genetic background delineates specific mechanisms of proliferation, survival or invasion/migration and regulates the response of melanoma cells to targeted therapy, we hypothesized with the possibility that mutations in BRAF or NRAS might affect melanoma cell response to chemotherapeutic agents.

\section{Results \\ NRAS mutant melanoma cells are less responsive to DTIC than BRAF mutant cells}

To address the potential influence of the genetic background on the response of melanoma cells to chemotherapeutic agents, we tested three different classes of DNA damaging agents: carmustine, cisplatin and DTIC in 9 NRAS mutant and 9 BRAF mutant melanoma cell lines (mutNRAS and mutBRAF cells; see Additional file 1: Table S1). When comparing the mean GI50 for all mutNRAS cells with that of mutBRAF cells, no statistically significant difference was observed when cells were treated with carmustine or cisplatin (Figure 1A-B). In contrast, mutNRAS cells were significantly more resistant to DTIC than mutBRAF cells $(\mathrm{p}<0.001$, Figure $1 C)$. In view of this result we decided to use TMZ as another triazene that acts on DNA through a mechanism identical to that of DTIC. Surprisingly, no significant difference was detected between the average GI50 for TMZ in mutBRAF and mutNRAS melanoma cells (Figure 1D).

\section{Light activated DTIC does not act through DNA alkylation} As mentioned TMZ undergoes spontaneous activation, whereas DTIC needs to be metabolized in the liver [13]. We had activated DTIC by exposure to white light, an alternative in vitro activation method previously described by others. Indeed we confirmed that light activation enhanced DTIC-mediated growth inhibition (Additional file 2: Figure S1A). To establish that this gives rise to a DNA alkylating agent, we quantified $O^{6}$ meG levels in DNA extracted from DTIC-treated cells and compared them with the levels in TMZ-treated cells. As expected, TMZ efficiently induced DNA methylation (Figure 2A). However, light activated DTIC even at high concentrations $(300 \mu \mathrm{M})$, was unable to induce any detectable DNA methylation in either mutBRAF or mutNRAS cell lines (Figure 2A). Furthermore, in line with the induction of DNA damage, Histone H2AX phosphorylation was detectable in TMZ treated cells, but no signal was observed in DTIC treated cells (Figure 2B). Accordingly, light activated DTIC failed to induce the activation of CHK-1 and CHK-2, kinases known to be activated by DNA damage (Additional file 2: Figure S1B).

Because MGMT activity is closely linked to MTIC mediated DNA damage, we determined the levels of MGMT in selected cell lines. We found no correlation between MGMT activity and NRAS or BRAF mutation status (Additional file 3: Table S2). However, as expected, MGMT expressing cells were significantly more resistant to TMZ (Figure $2 \mathrm{C}$ ), but 

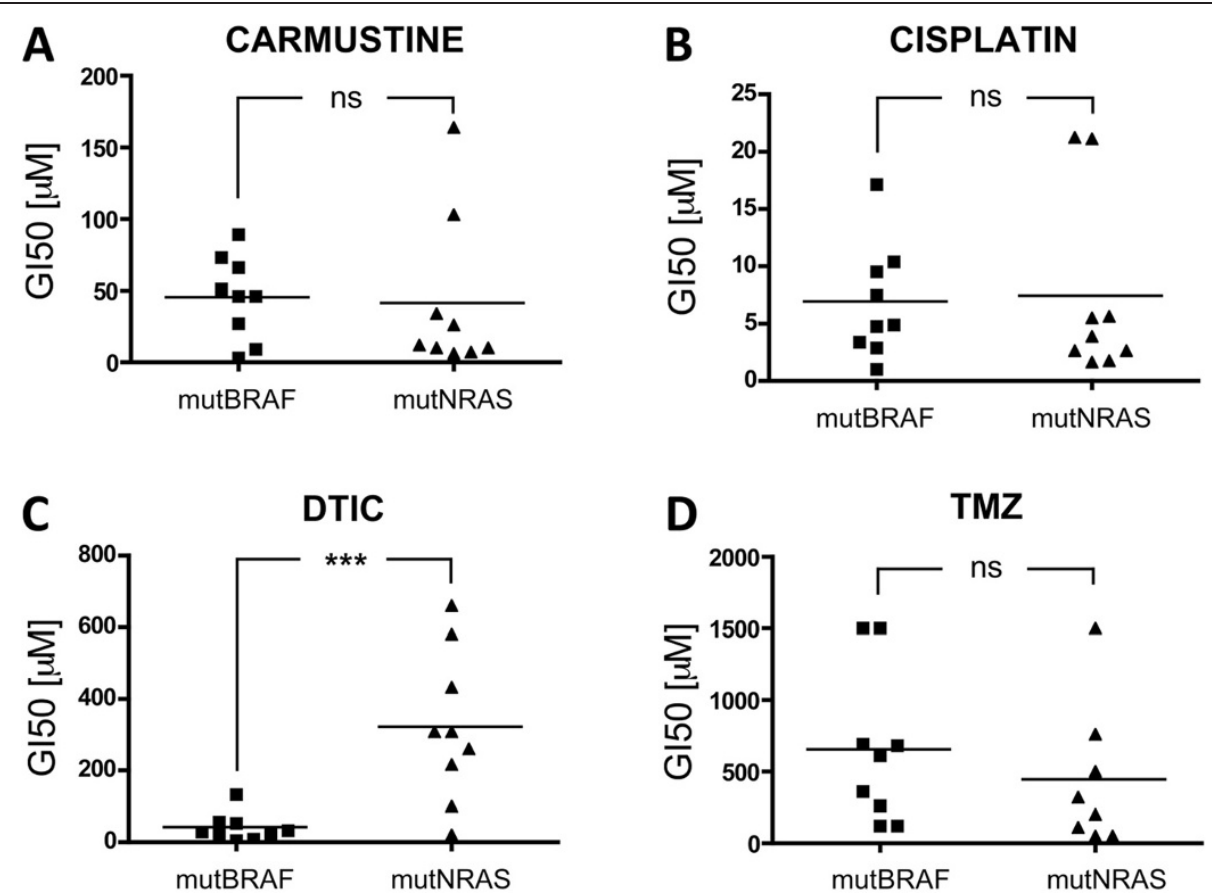

Figure 1 Mutation dependent response to alkylating agents. Nine melanoma cell lines with activating mutations in either BRAF or RAS were treated with serial increasing concentrations of $\mathbf{A}$, carmustine $\mathbf{B}$, cisplatin, $\mathbf{C}$, dacarbazine (DTIC) or $\mathbf{D}$, temozolomide (TMZ). After 5 days, cells were quantified using toluidine blue staining. Untreated cells were set $100 \%$ and the Gl50 for each drug was calculated as the mean of 3 independent measurements. Each point corresponds to an individual cell line. Student's t test compares the average GI50 for mutBRAF cell lines vs mutNRAS cell lines. $n s=$ not significant, ${ }^{* *} p=0.0006$ for DTIC MutBRAF vs mutRAS cells.

the average GI50 for DTIC was not significantly different between MGMT expressing and non-expressing cells (Figure 2D). Furthermore, A375P cells, which possess MGMT activity (Additional file 3: Table S2), are sensitized to TMZ by the MGMT inhibitor lomeguatrib, whereas lomeguatrib did not have any effect on DTIC treatment (Figure 2E). Similar results were obtained with other mutBRAF cells as well as with several mutNRAS cell lines including WM1361 (Figure 2E). These data indicate that light-activated DTIC, unlike TMZ, cannot induce DNA alkylation, and that the differential growth inhibitory activities observed in BRAF and NRAS mutant melanoma cells are due to alternative mechanisms.

\section{Light activated DTIC induces a G1 cell cycle arrest}

To gain more insight into the effect of DTIC on melanoma cell growth we analysed cell cycle progression in the presence of light activated DTIC and compared this to TMZ treatment. Exposure to TMZ for 72 hours led to a significant $\mathrm{G} 2 / \mathrm{M}$ arrest in both mutBRAF D10 cells and mutNRAS MM485 cells (Figure 3A and B). On the other hand, DTIC treatment with a concentration equivalent to the average GIC50 of all mutBRAF cells $(50 \mu \mathrm{M})$ led to block at the G1/S-transition in mutBRAF D10 cells (Figure 3A). Moreover, mutNRAS MM485 cells were largely unaffected by DTIC (Figure 3B). Similar results where obtained with other mutBRAF and mutNRAS melanoma cell lines (data not shown). We then analysed whether the accumulation in G1 was linked to a reduction in DNA synthesis and indeed, when we treated mutBRAF WM266-4 cells with DTIC for 24,48 or $72 \mathrm{~h}$, there was a progressive reduction in DNA synthesis, with a maximal inhibition of $65 \%$ at $72 \mathrm{~h}$ (Figure 3C).

\section{Hypoxanthine overcomes the DTIC mediated growth inhibition in melanoma cells}

The observation of a DNA synthesis phenotype was intriguing, because white light exposure of DTIC triggers its degradation to 2-aza-hypoxanthine (2-AzaHX) [22], which can interfere with DNA synthesis. Thereby 2AzaHX competes with the structurally related hypoxanthine (HX) as substrate for the hypoxanthine-guanine phosphoribosyl-transferase (HGPRT) in the purine salvage pathway (Figure 4A).

To assess the possibility that DTIC was transformed into 2-AzaHX, we analysed the UV absorption spectrum of DTIC and observed that light exposure of DTIC led to the formation of a metabolite with maximal UV absorption at 256-295 nm, similar to that of 2-AzaHX (Additional file 4: Figure S2).

Next we treated the melanoma cell lines with DTIC in the presence of $\mathrm{HX}$, which should compete with 2-AzaHX 

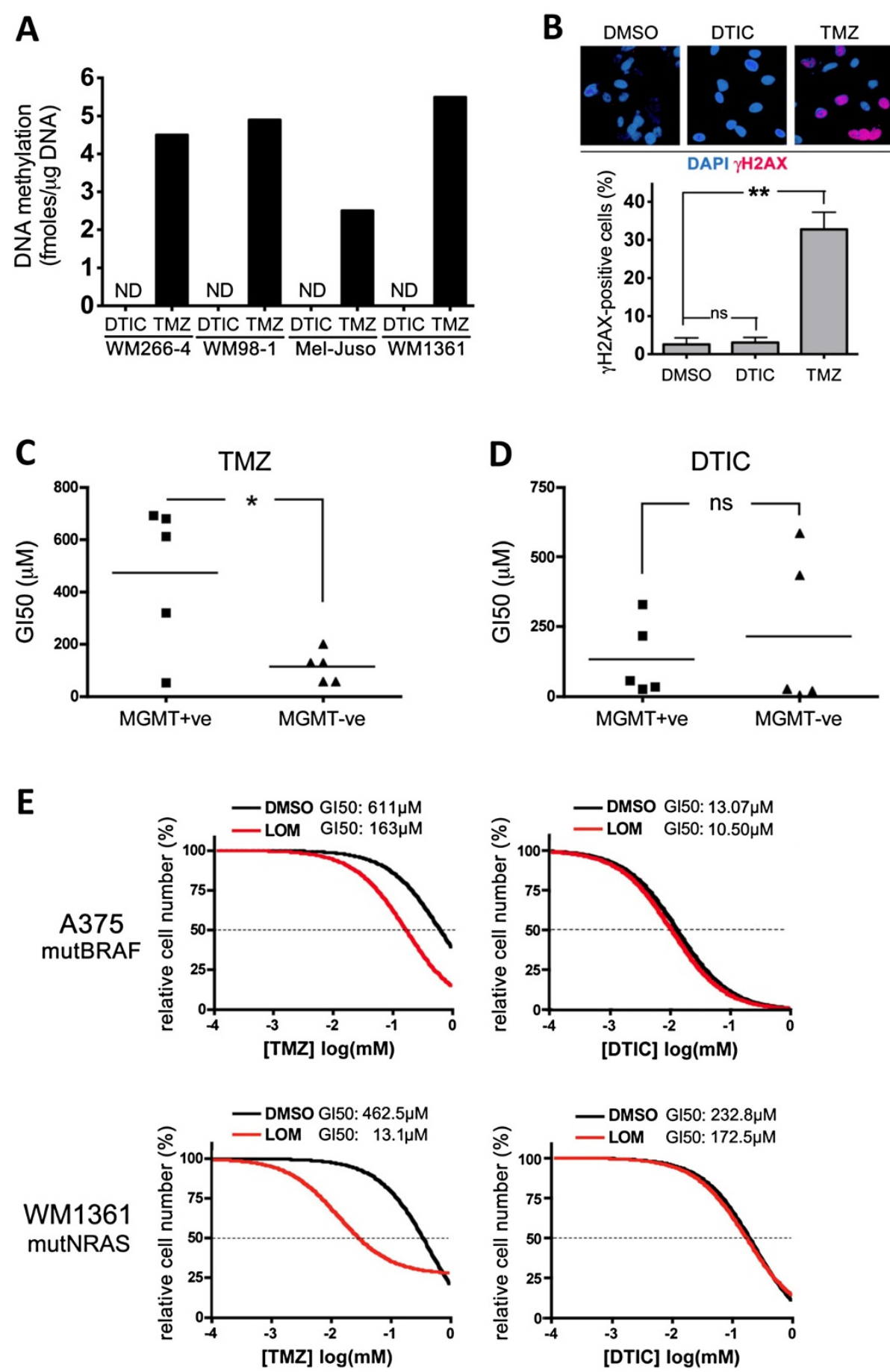

Figure 2 Light activated DTIC does not methylate DNA. A, Cells were treated with $300 \mu \mathrm{M}$ DTIC for $24 \mathrm{~h}$ or $1 \mathrm{mM}$ TMZ for 3 h, cells were harvested, genomic DNA purified and equal amounts of gDNA were used to determine the level of DNA methylation. ND: not detectable. B, Cells were treated with $300 \mu \mathrm{M}$ DTIC or $1 \mathrm{mM}$ TMZ for $24 \mathrm{~h}$ and stained for $\mathrm{YH} 2 \mathrm{AX}$ by immunofluorescence. C and $\mathbf{D}$, Cell lines were grouped upon their MGMT status (MGMT + ve=MGMT proficient; MGMT-ve=MGMT deficient), and the average GI50 for TMZ or DTIC was compared between both groups. Student's t test compares the average GI50 for MGMT + ve cells vs MGMT-ve cell. n.s.: not significant, ${ }^{*} p<0.05$. MGMT + ve cells are significantly more resistant to TMZ than MGMT-ve cells, $p=0.0116$. E, Dose response curve of cell survival to TMZ or DTIC in the absence (DMSO) or presence of the MGMT inhibitor lomeguatrib (LOM, $20 \mu \mathrm{M})$. A375 or WM1361 cells were treated with lomeguatrib $1 \mathrm{~h}$ before addition of different concentrations of DTIC or TMZ. After 5 days cells were stained with toluidine blue and quantified. DMSO treated cells were set as $100 \%$. The GI50 for each combinatorial treatment is shown. 


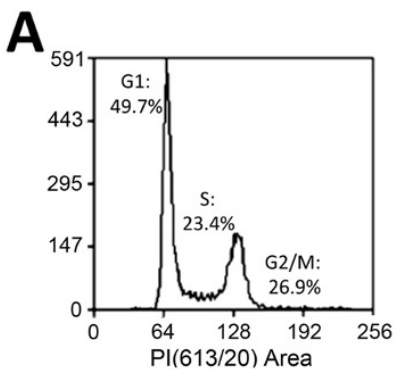

DMSO

B

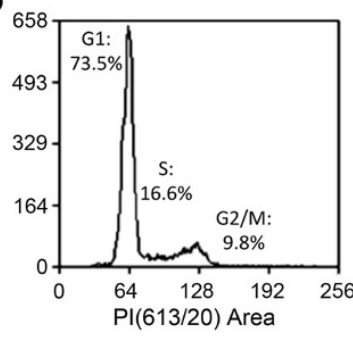

DMSO

\section{mutBRAF (D10)}

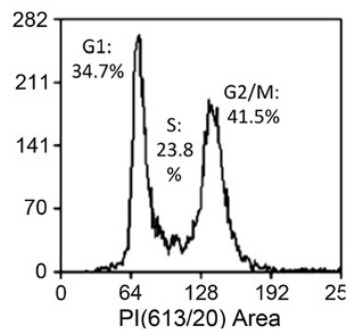

TMZ

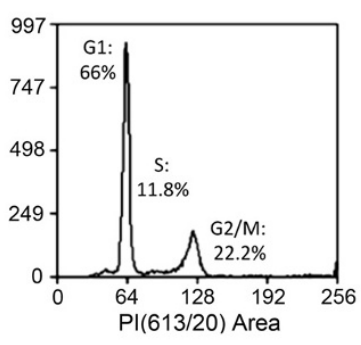

DTIC

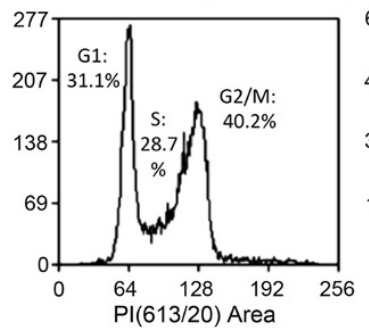

TMZ

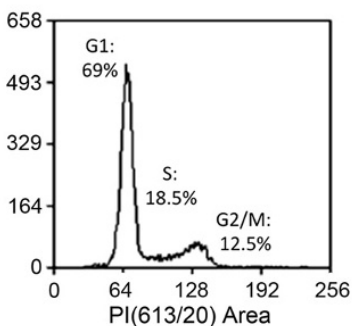

DTIC
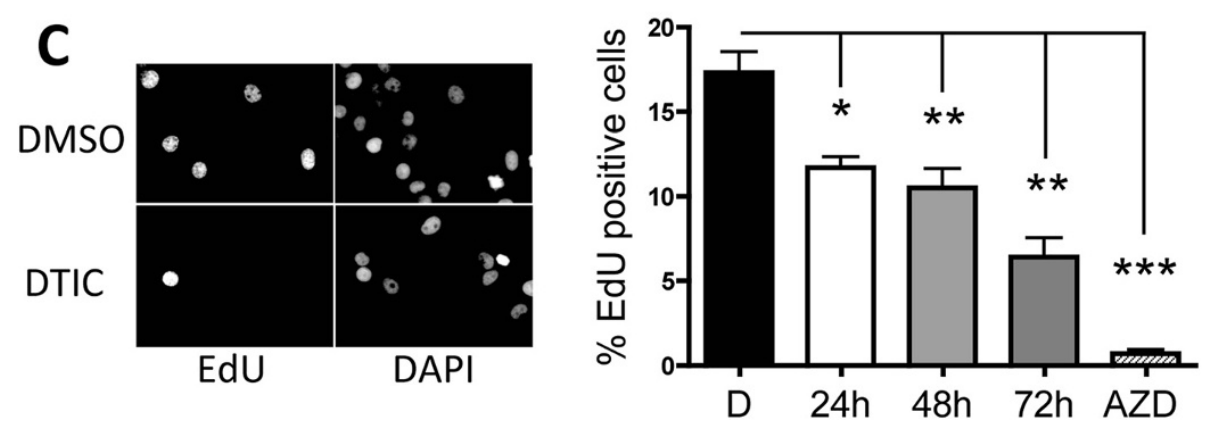

Figure 3 Light activated DTIC blocks melanoma cell proliferation. A and B, DNA content FACS analysis of D10 and MM485 cell lines treated with TMZ or DTIC for 72 hr. C, Proliferation assay. WM266-4 cells were treated with DTIC or the MEK inhibitor selumetinib and EdU incorporation was measured 24,48 or 72 hours after drug addition as indicated; untreated cells were set $100 \%$. One-way Anova was used to compare the effect of each treatment with untreated cells. ${ }^{*} p<0.05,{ }^{* *} p<0.01,{ }^{* * *} p<0.001$.

for HGPRT binding and prevent its inhibitory effect. Indeed, the addition of HX significantly overcame the effects of DTIC in WM266-4 cells, increasing the GI50 to DTIC by almost 15 -fold (Figure 4B). Moreover, in the presence of $\mathrm{HX}$, the average GI50 for DTIC in mutBRAF cells was comparable to that of mutNRAS melanoma cells in the absence of HX $(\sim 300 \mu \mathrm{M}$, Figure $4 \mathrm{C})$. Notably, when mutNRAS melanoma cells were treated with $300 \mu \mathrm{M}$ DTIC HX addition also reverted the growth effects (Figure 4D), suggesting that DTIC inhibits the purine salvage pathway also in these cells. In support of this finding, the addition of 5 '-guanosine monophosphate (GMP) or guanosine (GR) rescued the inhibitory effect of DTIC in mutBRAF and mutNRAS cells (Figure 4E). These results support the hypothesis that the growth inhibitory effect of light activated DTIC is mediated by its degradation to 2-AzaHX, which inhibits the purine salvage pathway.

\section{NRAS mutant melanoma cells display higher nucleotide} salvage pathway activity than BRAF mutant cells

Nucleotide salvage pathways are crucial for efficient DNA synthesis, particularly in fast dividing cells. In line with this, we found increased expression of the key enzymes of nucleotide salvaging HGPRT (HPRT1), thymidine kinase (TK1) and APRT in melanoma (primary and metastatic) 
A<smiles>O=c1[nH]cnc2[nH]cnc12</smiles>

Hypoxanthine

$\mathrm{HX}$<smiles></smiles>

2-Azahypoxanthine

2-AzaHX

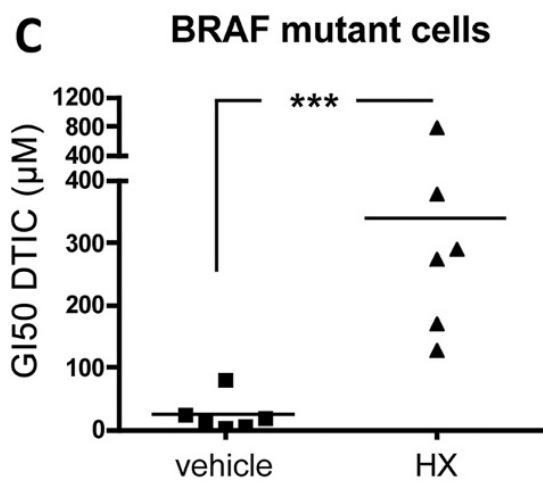

B

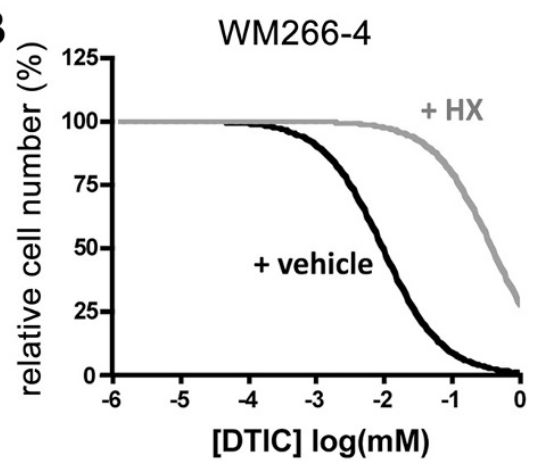

D

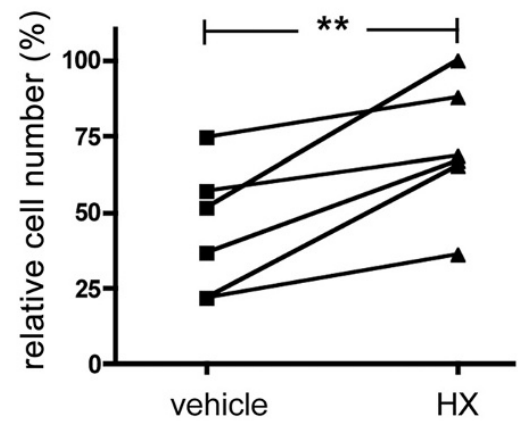

E

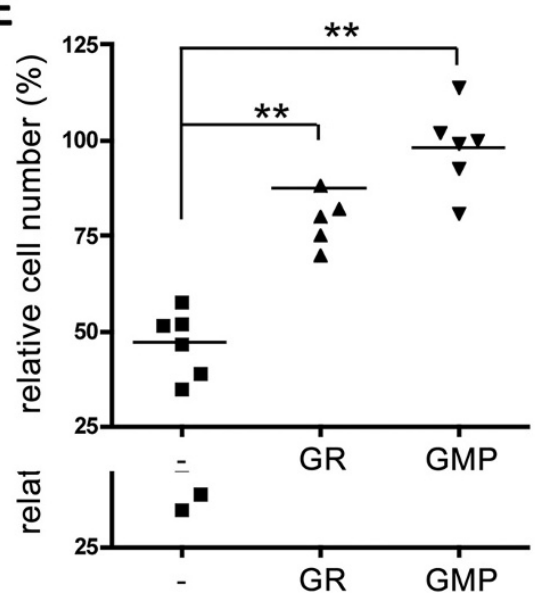

Figure 4 The DTIC inhibitory effect is overcome by hypoxanthine. A, Structure of hypoxanthine $(H X)$ and 2-azahypoxanthine (2-AzaHX). B, Dose response curve of WM266-4 cell survival to DTIC in the absence (vehicle) or presence of $100 \mu \mathrm{M}$ hypoxanthine (HX). Untreated cells were set as 100\%. C, Graph showing the GI50 for DTIC of six mutBRAF melanoma cell lines treated as in B. The average GI50 for cells co-treated with HX (336.5 $\mu \mathrm{M})$ was significantly higher than for vehicle-treated cells $(22.89 \mu \mathrm{M})$, as determined by Student's $t$ test. ${ }^{* *} p=0.0046$. D, Quantification of cell survival of five mutRAS melanoma cell lines upon treatment with $300 \mu \mathrm{M}$ DTIC in the absence (vehicle) or presence of $100 \mu \mathrm{M}$ hypoxanthine (HX). E, Cell survival of 3 mutBRAF and 3 mutNRAS cell lines treated with $50 \mu \mathrm{M}$ or $300 \mu \mathrm{M}$ DTIC respectively in the absence (-) or presence of $100 \mu \mathrm{M}$ Guanosine (GR) or 2'5'-GMP (GMP).

compared to normal skin and benign nevi ( Figure 5A). We then argued that despite the general increased salvage activities in melanoma cells, mutNRAS cells might exhibit and even higher efficiency of salvage pathway usage than mutBRAF cells, which would render them resistant to the inhibitory effect of 2-AzaHX. To test this hypothesis we cultured melanoma cell lines in the presence of an inhibitor of de novo DNA synthesis, aminopterin. Under these conditions cell growth is mainly driven via nucleotide salvage pathways, which is fuelled by the addition of the 


\section{A}

Talantov melanoma cell line data set

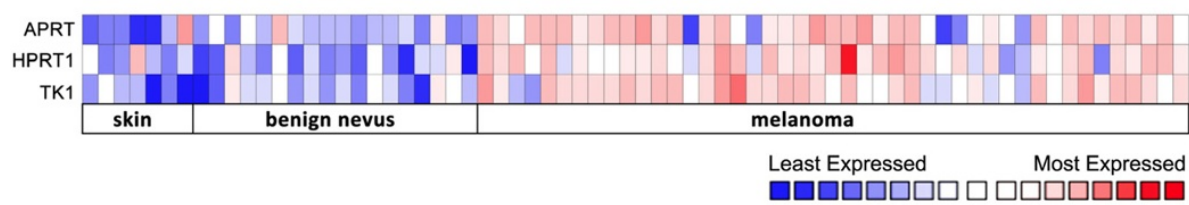

B

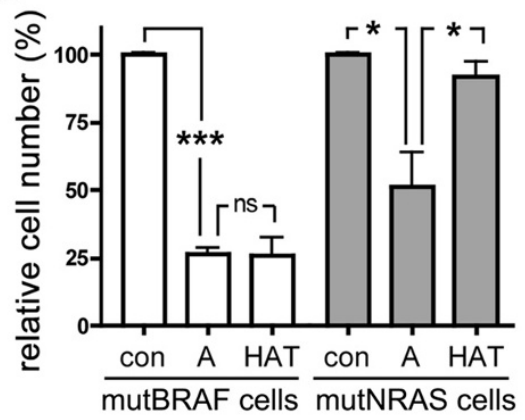

D
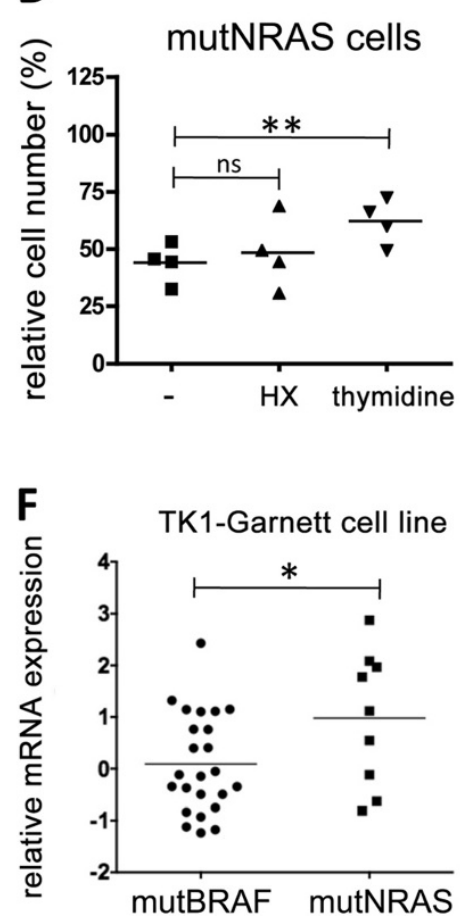

C

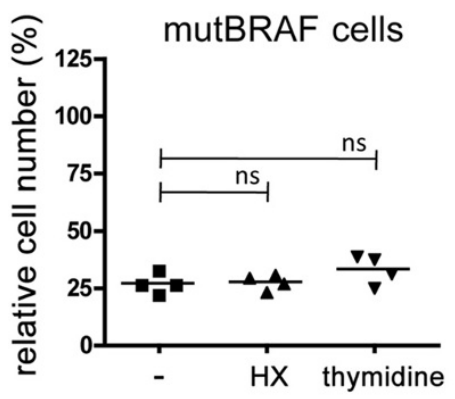

$\mathbf{E}$
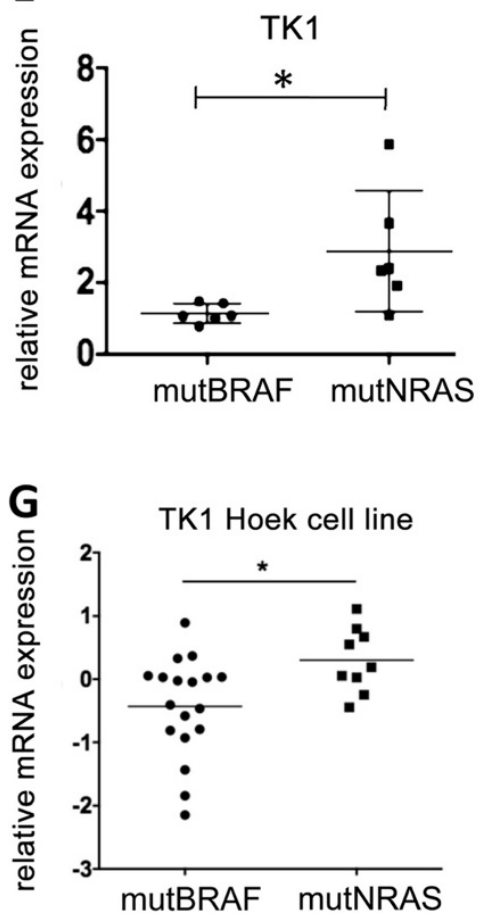

Figure 5 mutNRAS melanoma cells possess increased thymidine salvage capacity. A, Heat map of expression profile of APRT, HPRT1 and TK1 genes in normal skin, benign nevus and melanoma in a data set obtained from Oncomine [24]. B, Four mutBRAF and mutNRAS melanoma cell lines were treated with $0.4 \mu \mathrm{M}$ aminopterine in the absence (A) or presence of hypoxanthine and thymidine (HAT). After 3 days cells were fixed, stained with toluidine blue and surviving fractions were quantified. C, Four mutBRAF or D, mutNRAS cell lines were grown in normal medium supplemented with $0.4 \mu \mathrm{M}$ aminopterin in the presence or absence of $100 \mu \mathrm{M} \mathrm{HX}$ or $16 \mu \mathrm{M}$ thymidine, as indicated. After 3 days the survival fraction was determined. Cells cultured in normal medium were set as 100\% survival. E-G, Comparison of thymidine kinase (TK1) mRNA expression in mutBRAF and mutNRAS melanoma cell lines (as assessed by q-RT-PCR) in our panel of melanoma cell lines or in two independent data sets deposited in Oncomine [25,26]. ${ }^{*} p<0.05,{ }^{* *} p<0.01,{ }^{* * *} p<0.001$. 
supplements HX and thymidine 005B [23]. In the presence of aminopterin, the growth of all cell lines was significantly reduced (Figure 5B), indicating that de novo DNA synthesis is required for cell growth. However, whereas the addition of $\mathrm{HX}$ and thymidine almost completely rescued the growth of mutNRAS cell lines, mutBRAF cell lines did not show an increase in cell growth (Figure 5B). This suggested that although mutBRAF cells use salvage pathways for cell growth when de novo synthesis is inhibited $(25 \%$ cell growth after 3 days of inhibition), the efficiency of this alternative DNA synthesis route is much lower in these cells than in mutNRAS cells.

We next quantified the individual effects of adding HX and thymidine as salvage substrates for HGPRT and thymidine kinase, respectively. Interestingly, when the de novo synthesis was inhibited addition of HX alone did not enhance cell growth in mutNRAS and mutBRAF cells (Figure $5 \mathrm{C}$ and $\mathrm{D}$ ), suggesting that under these conditions the cells might be using endogenously produced guanine as an alternative substrate [27]. On the other hand, mutNRAS cells were significantly more efficient than mutBRAF cells in utilising thymidine to counteract the growth inhibitory effect of de novo synthesis inhibition (Figure 5C and D).

Thymidine is the substrate of TK1 in the pyrimidine salvage pathway and our data suggested that TK1 activity is increased in mutNRAS cells. Indeed, we found a significant overexpression of TK1 in mutNRAS cells compared to mutBRAF cells in our panel of melanoma cell lines (Figure 5E). This finding was supported by two independent datasets [25,26] analysed in Oncomine (Figure 5F and G).

NRAS mutant melanoma cells are more resistant to DNA de novo synthesis inhibitors than BRAF mutant cells

Our data show that in mutNRAS melanoma cells elevated TK1 activity contributes to enhanced pyrimidine salvaging. However, the inhibitory effect of 2-AzaHX is on the purine salvage pathway, where after conversion into 2-Aza-inositol monophosphate (2-AzaIMP) it suppresses IMP dehydrogenase (IMPDH) (Figure 6A). Thus, the difference in the response of mutNRAS and mutBRAF cells to DTIC could be based on differences in IMPDH. Indeed, mutNRAS cells were significantly more resistant to two IMPDH inhibitors, Mycophenolic Acid (MPA) and AVN944, compared to mutBRAF cells (Figure 6B). IMPDH expression levels did not differ in the individual cell lines (data not shown), indicating that the resistance in mutNRAS cells is not due to higher IMPDH expression levels.

In summary, mutNRAS melanoma cells are more efficient in nucleotide salvaging than mutBRAF melanoma cells, which is at least part due to enhanced TK1 expression and IMPDH activity (Figure 6A). This finding suggests that mutNRAS melanoma cells would be more resistant to drugs targeting DNA de novo synthesis than mutBRAF cells. Indeed, when we determined the GI50 of our panel of melanoma cells for the DHFR inhibitors aminopterin, pyrimethamine and methrotrexate, we found that mutNRAS cells were significantly more resistant than mutBRAF cells (Figure 6C). Moreover, when we analysed a dataset derived from a drug screen using a large panel of cancer cell lines [25], we found not only that NRAS mutant melanoma cell lines were more resistant to pyrimethamine than BRAF mutant melanoma cells (Figure 6D), but that independently of cancer type, mutRAS cancer cells were significantly more resistant to pyrimethamine than mutBRAF cells (Figure 6E).

\section{Discussion}

The aim of this study was to determine whether the mutational status of melanoma cells would correlate with their response to chemotherapeutic agents. Our results demonstrate that in melanoma the presence of mutually exclusive BRAF and NRAS mutations has no influence on the response to DNA alkylating agents such as TMZ. Similar results considering BRAF or RAS mutation status are found in a large data set containing drug-treatment data from 732 cancer cell lines of different origin [25]. Thus, it appears that mutBRAF and mutNRAS share common mechanisms of resistance to methylating agents such as drug efflux, or deregulation of pro-apoptotic or DNA repair pathways.

Surprisingly, we found that mutBRAF and mutNRAS cells respond very differently to light activated DTIC. Activation of DTIC by exposure to white light has been described to recapitulate its chemotherapeutic activity in vitro $[28,29]$ but our results provide experimental evidence that the toxic effect described for light activated DTIC is independent of DNA methylation. Through DNA alkylation assays and combinatorial treatments using DTIC and a MGMT inhibitor we provide clear evidence that light exposure does not transform DTIC into a DNA methylating agent, but rather an inhibitor of DNA synthesis. This finding is of major importance considering that light activation of DTIC has been extensively used to study the mechanisms underlying its cytotoxic effects as well as leading to acquired resistance in patients [28-31]. In this context it is important to mention that wild type BRAF melanoma cells that had been selected for resistance to light activated DTIC in vitro exhibited increased tumour growth in vivo, a phenotype that correlates well with enhanced DNA synthesis activity [32]. Most strikingly these resistant cells and tumours displayed hyper-activation of the MAP-kinase pathway, resulting in increased IL8 and VEGF expression $[28,32]$. The fact that we now show that light activated DTIC inhibits nucleotide synthesis, most probably by inhibiting IMPDH, suggests a novel link between DNA synthesis pathways and MAP-kinase signalling. 

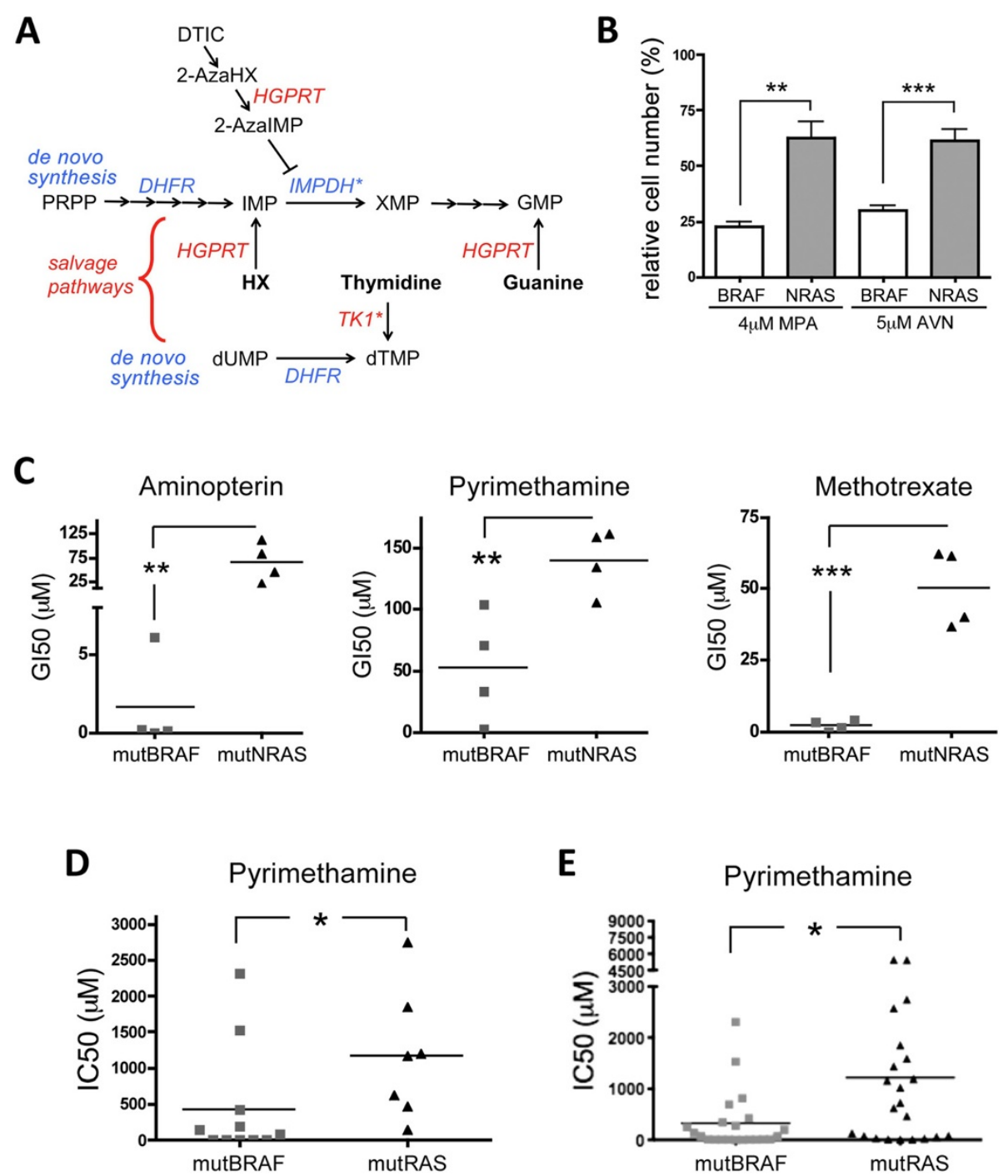

Figure 6 mutRAS melanoma cells are more resistant to DHFR targeting drugs. A, Schematic of nucleotide de novo synthesis and salvage pathways. The asterisks indicate increased activity (IMPDH) or expression (TK1) in mutNRAS cells. B, Average survival of 4 mutBRAF or mutNRAS cell lines after treatment with $4 \mu \mathrm{M}$ Mycophenolic Acid (MPA) or $5 \mu \mathrm{M}$ AVN499. ${ }^{* *} p=0.01,{ }^{* * *} p<0.001$. C, Melanoma cell lines with activating mutations in either BRAF or NRAS were treated with serial increasing concentrations of aminopterin, pyrimethamine or methotrexate. After 3 days, cells were fixed, stained and the GI50 for each drug was calculated. Student's t test (one-tailed) compares the average GI50 for mutBRAF cell lines vs mutNRAS cell lines. Aminopterin: ${ }^{* *} p=0.0092$; Pyrimethamine: ${ }^{* *} p=0.0071$; Methotrexate: ${ }^{* *} p=0.0002$. D, Comparison of the IC50 for dypirimethamine of 11 mutBRAF and 7 mutRAS melanoma cell lines, as determined by MTS assay in [25]. * $p=0.0393$. E, Graph comparing the IC50 for dypirimethamine between 22 mutBRAF and 22 mutNRAS cell lines from different tumor types [25]. Student's $t$ test,

two-tailed: ${ }^{*} p=0.0184$.

Our results indicate that non-metabolically activated DTIC mediates its effects through 2-AzaHX. Strikingly, there is evidence that metabolic activation of DTIC in patients is inefficient and that, shortly after DTIC isolated limb perfusion, significant amounts of 2-AzaHX can be detected in the bloodstream and urine of patients [33]. Thus it is possible that 2-AzaHX could contribute to the DTIC-dependent toxicity, although it is well established that the anti-tumour activity of DTIC is mainly the result of DNA methylation [13].
In this context it is important to mention that conversion of $2 \mathrm{AzaHX}$ by HGPRT to 2-AzaIMP is able to inhibit IMPDH $[34,35]$. We found that mutNRAS melanoma cells are significantly more resistant to two bona fide IMPDH inhibitors (MPA and AVN944), suggesting that in NRAS mutant cells IMPDH activity or its downstream signalling is elevated. This, together with the fact that mutNRAS cells express higher levels of TK1 and consequently are more effective in using thymidine for DNA synthesis, provides strong evidence 
that mutNRAS melanoma cells are significantly more efficient in nucleotide salvaging.

Increased $I M P D H 2$ expression in cancer cells has been linked to resistance to methotrexate in osteosarcoma, colorectal and erythroleukemia cells [36-38]. However, although IMPDH2 is overexpressed in melanoma compared to benign melanocytic lesions (not shown), its expression did not differ in mutNRAS and mutBRAF melanoma cells. Therefore, the difference in the response to IMPDH inhibitors rather suggests that IMPDH activity or its downstream signalling is regulated differently in mutNRAS compared to mutBRAF cells. Apart from IMPDH, we also show that thymidine can compensate for DHFR inhibition in resistant mutNRAS cells, which express higher levels of TK1. Whether elevated TK1 expression is directly regulated by NRAS is not yet known, but it will be crucial to identify the underlying mechanism. Importantly, we did not find differences in TK2 expression between mutNRAS and mutBRAF cells (not shown), which is maybe not surprising considering the more ubiquitous role of TK2 [39].

Historically antifolate drugs such as methotrexate or edatrexate have shown very little activity in clinical trials with melanoma patients although these trials were performed before the discovery of BRAF and RAS as drivers of melanomagenesis [40,41]. The lack of response in melanoma patients can be explained by several mechanisms of resistance such as melanosomal sequestration of drugs, the upregulation of both DHFR and the pro-survival transcription factor MITF in response to MTX, or the E2F and Chk1 mediated effects, as recently described [42-45]. Despite the inherent capacity to resist any chemotherapy our data suggest that stratifying patients according to their BRAF/RAS mutation status could lead to better responses to antifolate based therapies.

Importantly, our findings suggest that the correlation between NRAS and BRAF mutations and their differential response to antifolate drugs might apply to other cancer types. Therefore, in cancer types where antifolate based therapies contribute to achieve clinical responses in RAS patients (e.g. colorectal carcinoma) [46], it would be interesting to assess whether mutBRAF patients show even improved responses. If that were the case it would open the possibility to use mutational status as a predictor of patient response. In summary, our findings identify the mutually exclusive NRAS and BRAF mutation status as possible predictive marker for the response to DNA synthesis inhibitors such as antifolate drugs in melanoma patients.

\section{Conclusions}

In summary in this study we demonstrate that activation of DTIC with white light does not result in a methylating agent but in to an inhibitor of purine synthesis. We show that RAS mutant melanoma cells are more resistant to drugs affecting DNA synthesis than BRAF mutant cells. Our data suggests that, the increased resistance to de novo DNA synthesis inhibitors found in RAS mutant cells is due to a superior capacity to salvage DNA. Notably our results suggest the possibility that the correlation between RAS and BRAF mutations and the response to antifolate drugs might be relevant in other cancer types although further efforts to confirm this hypothesis are warranted.

\section{Materials and methods \\ Cell culture}

Nine mutant BRAF cell lines and nine mutant NRAS cell lines were used in the study (Additional file 1: Table S1). These cells were a kind gift from Dr. Richard Marais and Dr. Adam Hurlstone. Cell stocks were expanded, frozen, and kept in liquid nitrogen. New aliquots were thawed every 5-7 weeks. Cells were cultured in Dulbecco's Modified Eagle's Medium (DMEM) (SIGMA) or in RPMI-164 medium (RPMI) (SIGMA) as previously indicated, supplemented with $0.5 \%$ penicillin and streptomycin (SIGMA) and $10 \%$ bovine calf serum (PAA, Yeovil, UK). Cells were grown at $37^{\circ} \mathrm{C}$ in a $5 \% \mathrm{CO}_{2}$ environment.

\section{Reagents}

HAT supplement (50X) was from Sigma. Dacarbazine, carmustine, cisplatin and temozolomide and lomeguatrib were from SIGMA. Hypoxanthine, guanosine and 5 '-guanosine monophosphate were from Sigma. AZD6244 was from Selleck Chemicals, Newmarket, UK. Mycophenolic Acid and AVN944 were from Sigma and ChemieTek respectively. Aminopterin, pyrimethamine and amethopterin (methotrexate) were from Sigma. All drugs were dissolved in dimethylsulfoxide (DMSO) and, apart from dacarbazine, directly added to cell in culture at the indicated concentrations. Prior to addition onto cells DTIC was exposed to white light for $1 \mathrm{~h}$, as previously described [28,30].

\section{Determination of MGMT activity}

Melanoma cell free extracts prepared from $10^{6}$ cells were analysed for MGMT activity using calf thymus DNA methylated in vitro with $\mathrm{N}$-nitroso- $\mathrm{N}-\left[{ }^{3} \mathrm{H}\right]$-methylurea $(\sim 20 \mathrm{Ci} /$ $\mathrm{mmol}$ ) as the substrate [47]. MGMT activity was expressed as $\mathrm{fmol} / \mu \mathrm{g}$ DNA to avoid the possible effect of variable protein content on apparent MGMT activity expressed per unit protein [48]. No significant differences in the study results were noted when MGMT activity was expressed per unit protein. Results are the mean of quadruplicate determinations for each sample. Cell free extracts prepared from the human breast cancer cell line MCF-7 were assayed for MGMT activity as a positive control.

\section{Determination of 06-methylguanine levels in DNA}

O6-methylguanine $\left(O^{6}\right.$-meG) in DNA was quantified using a modification of the standard MGMT activity assay procedure [49]. Increasing amounts of the DNA samples 
were pre-incubated with a standard amount of purified recombinant human MGMT [50] and residual activity was then determined. $\mathrm{O}^{6}$-meG in DNA stoichiometrically inactivates MGMT. Thus the amount of $O^{6}-\mathrm{meG}$ in the DNA sample equals the amount of inactivation of the purified MGMT.

\section{Determination of GI50}

To determine the drug concentration necessary to inhibit cell growth by $50 \%$ (GI50), 2000 cells per well were plated in 96 well plates (Corning). After 24 hours, drugs were added in triplicates in serial 1:3 dilutions. In experiments where cells were co-treated with the MGMT inactivating agent lomeguatrib, the drug was added 1 hour before the addition of serial dilutions of DTIC or TMZ. After 3 or 5 days cells were washed with PBS and simultaneously fixed and stained for 1 hour with 4\% Formaldehyde (Fisher Scientific) and $0.5 \%$ Toluidine Blue (Fluka Analytical) in PBS. Plates were washed, dried and the dye was solubilized with $1 \%$ Sodium dodecyl sulfate (SDS) (Fisher Scientific) in PBS. Finally, a spectrophotometer (BIO-TEK ${ }^{\oplus}$, NorthStar Scientific) was used to measure the O.D. and GI50 values were calculated using the GraphPad Prism software (GraphPad Software, 4.0a).

\section{Databases}

To study the expression profile of APR1, HPRT1 and TK1 genes in human melanoma versus normal skin or benign nevus, and to compare TK1 expression between mutBRAF and mutRAS human melanoma cell lines we used Oncomine Cancer Microarray database (http:// www.oncomine.org/).

\section{RNA isolation and qPCR analysis}

RNA was isolated with TRIZOL $^{\circ}$ and selected genes were amplified by quantitative real time PCR using SYBR green (Qiagen, Valencia, CA, USA).

Primers sequences were:

TK1:

Forward: 5' -TGGCTGTCATAGGCATCGAC-3', Reverse: 5' -CCAGTGCAGCCACAATTACG-3'

BETA-ACTIN:

Forward: 5' -GCAAGCAGGAGTATGACGAG-3', Reverse: 5' -CAAATAAAGCCATGCCAATC-3'

\section{EdU incorporation assays}

Cells were labelled with $10 \mu \mathrm{M}$ EdU (Invitrogen) for $4 \mathrm{~h}$ before they were formalin fixed and processed following the manufacturer's instructions. Stained cells were analysed using a BDpathway 855 Bioimager.

\section{FACS analysis}

100000 cells were treated as indicated, fixed in ice-cold 80\% ethanol. Cells were then washed in PBS and incubated in a solution containing PBS, RNase $\mathrm{A}$ and Propidium Iodide (SIGMA) at $37^{\circ} \mathrm{C}$ for 1 hour. The analysis was performed using FACS Calibur (Becton Dickinson).

\section{Statistical analysis}

Unless indicated otherwise, data are from assays performed in triplicate, with error bars to represent standard deviations or errors from the mean. Statistics used were: predominately Student t-test and One-way ANOVA with Dunnett's Multiple Comparison Test performed using GraphPad Prism version 4.00 for Mac OS, GraphPad Software, San Diego California USA, www.graphpad.com.

\section{Additional files}

Additional file 1: Table S1. List of melanoma cell lines used in this study, grouped upon the presence of activating mutations in either BRAF or RAS.

Additional file 2: Figure S1. A, DTIC exposure to white light increases DTIC' inhibitory effect.

Additional file 3: Table S2. MGMT status of a panel of 10 melanoma cell lines MGMT activity in 5 mutBRAF and 5 mutNRAS melanoma cell lines. Exponentially growing cells were harvested, gDNA was isolated and the presence of MGMT activity as well as the concentration of MGMT was determined. N.d.: not detectable.

Additional file 4: Figure S2. 2-Azahypoxanthine and light activated DTIC show similar UV absorbance profiles.

\section{Abbreviations}

DTIC: Dacarbazine; TMZ: Temozolomide; MGMT: Methylguanine-DNA-methyltransferase; HX: Hypoxhantine; GMP: 5'-guanosine monophosphate; GR: Guanosine; IMPDH: Inosine-5'-monophosphate dehydrogenase; HGPRT: Hypoxanthine-guanine phosphoribosyltransferase.

\section{Competing interests}

The authors declare no conflict of interest.

\section{Authors' contributions}

IA conceived the study, designed and carried out most of the experiments, analysed the data and wrote the manuscript. IG and OE carried out the experiments to assess the Gl50 of every alkylating agent and the effect on the cell cycle. JF was in charge of q-RT-PCR experiments. GPM carried out DNA methylation and MGMT activity assays and helped write the manuscript. CW conceived and coordinated the study, and wrote the manuscript. All authors read and approved the final manuscript.

\section{Acknowledgements}

This study was supported in part by the National Science Foundation of China and Cancer Research UK (C11591/A10202). The funders had no role in study design, data collection and analysis, decision to publish, or preparation of the manuscript.

\section{Author details}

${ }^{1}$ Manchester Cancer Research Centre, The University of Manchester, Michael Smith Building, Oxford Road, Manchester M13 9PT, UK. ºncology area, Biodonostia Research Institute, Calle Doctor Begiristain, San Sebastian 20014 Spain. ${ }^{3}$ Division of Hepatology and Gastroenterology, Biodonostia Research Institute, Calle Doctor Begiristain, San Sebastian 20014, Spain. ${ }^{4}$ Centre for Occupational and Environmental Health, The University of Manchester, Stopford Building, Oxford Road, Manchester M13 9PL, UK.

Received: 22 November 2013 Accepted: 11 June 2014 Published: 19 June 2014 


\section{References}

1. Davies H, Bignell GR, Cox C, Stephens P, Edkins S, Clegg S, Teague J, Woffendin H, Garnett MJ, Bottomley W, Davis N, Dicks E, Ewing R, Floyd Y, Gray K, Hall S, Hawes R, Hughes J, Kosmidou V, Menzies A, Mould C, Parker A, Stevens C, Watt S, Hooper S, Wilson R, Jayatilake H, Gusterson BA, Cooper C, Shipley J, et al: Mutations of the BRAF gene in human cancer. Nature 2002, 417:949-954.

2. Wellbrock $C$, Hurlstone A: BRAF as therapeutic target in melanoma Biochem Pharmacol 2008, 80:561-567.

3. Arozarena I, Sanchez-Laorden B, Packer L, Hidalgo-Carcedo C, Hayward R, Viros A, Sahai E, Marais R: Oncogenic BRAF induces melanoma cell invasion by downregulating the CGMP-specific phosphodiesterase PDE5A. Cancer Cell 2011, 19:45-57.

4. Belden S, Flaherty KT: MEK and RAF inhibitors for BRAF-mutated cancers. Expert Rev Mol Med 2012, 14:e17.

5. Chapman PB, Hauschild A, Robert C, Haanen JB, Ascierto P, Larkin J, Dummer R, Garbe C, Testori A, Maio M, Hogg D, Lorigan P, Lebbe C, Jouary T, Schadendorf D, Ribas A, O'Day SJ, Sosman JA, Kirkwood JM, Eggermont AM, Dreno B, Nolop K, Li J, Nelson B, Hou J, Lee RJ, Flaherty KT, McArthur GA, BRIM-3 Study Group: Improved survival with vemurafenib in melanoma with BRAF V600E mutation. N Engl I Med 2011, 364:2507-2516.

6. Flaherty KT, Infante JR, Daud A, Gonzalez R, Kefford RF, Sosman J, Hamid O, Schuchter L, Cebon J, Ibrahim N, Kudchadkar R, Burris HA 3rd, Falchook G, Algazi A, Lewis K, Long GV, Puzanov I, Lebowitz P, Singh A, Little S, Sun P, Allred A, Ouellet D, Kim KB, Patel K, Weber J: Combined BRAF and MEK inhibition in melanoma with BRAF V600 mutations. N Engl J Med 2012, 367:1694-1703.

7. Sosman JA, Kim KB, Schuchter L, Gonzalez R, Pavlick AC, Weber JS, McArthur GA, Hutson TE, Moschos SJ, Flaherty KT, Hersey P, Kefford R, Lawrence D, Puzanov I, Lewis KD, Amaravadi RK, Chmielowski B, Lawrence HJ, Shyr Y, Ye F, Li J, Nolop KB, Lee RJ, Joe AK, Ribas A: Survival in BRAF V600-mutant advanced melanoma treated with vemurafenib. N Engl J Med 2012, 366:707-714.

8. Wagle N, Emery C, Berger MF, Davis MJ, Sawyer A, Pochanard P, Kehoe SM Johannessen CM, Macconaill LE, Hahn WC, Meyerson M, Garraway LA: Dissecting therapeutic resistance to RAF inhibition in melanoma by tumor genomic profiling. I Clin Oncol 2011, 29:3085-3096.

9. Joseph EW, Pratilas CA, Poulikakos PI, Tadi M, Wang W, Taylor BS, Halilovic E, Persaud Y, Xing F, Viale A, Tsai J, Chapman PB, Bollag G, Solit DB, Rosen N: The RAF inhibitor PLX4032 inhibits ERK signaling and tumor cell proliferation in a V600E BRAF-selective manner. Proc Natl Acad Sci U S A 2010, 107:14903-14908.

10. Solit DB, Garraway LA, Pratilas CA, Sawai A, Getz G, Basso A, Ye Q, Lobo JM, She Y, Osman I, Golub TR, Sebolt-Leopold J, Sellers WR, Rosen N: BRAF mutation predicts sensitivity to MEK inhibition. Nature 2006, 439:358-362.

11. Heidorn SJ, Milagre C, Whittaker S, Nourry A, Niculescu-Duvas I, Dhomen N, Hussain J, Reis-Filho JS, Springer CJ, Pritchard C, Marais R: Kinase-dead BRAF and oncogenic RAS cooperate to drive tumor progression through CRAF. Cell 2010, 140:209-221.

12. Mouawad R, Sebert M, Michels J, Bloch J, Spano JP, Khayat D: Treatment for metastatic malignant melanoma: old drugs and new strategies. Crit Rev Oncol Hematol 2010, 74:27-39.

13. Marchesi F, Turriziani M, Tortorelli G, Avvisati G, Torino F, De Vecchis $L$ : Triazene compounds: mechanism of action and related DNA repair systems. Pharmacol Res 2007, 56:275-287.

14. Middleton MR, Grob JJ, Aaronson N, Fierlbeck G, Tilgen W, Seiter S, Gore M, Aamdal S, Cebon J, Coates A, Dreno B, Henz M, Schadendorf D, Kapp A, Weiss J, Fraass U, Statkevich P, Muller M, Thatcher N: Randomized phase III study of temozolomide versus dacarbazine in the treatment of patients with advanced metastatic malignant melanoma. J Clin Oncol 2000, 18:158-166.

15. Friedman HS, Kerby T, Calvert H: Temozolomide and treatment of malignant glioma. Clin Cancer Res 2000, 6:2585-2597.

16. Hauschild A, Grob JJ, Demidov LV, Jouary T, Gutzmer R, Millward M, Rutkowski P, Blank CU, Miller WH Jr, Kaempgen E, Martín-Algarra S, Karaszewska B, Mauch C, Chiarion-Sileni V, Martin AM, Swann S, Haney P, Mirakhur B, Guckert ME, Goodman V, Chapman PB: Dabrafenib in BRAF-mutated metastatic melanoma: a multicentre, open-label, phase 3 randomised controlled trial. Lancet 2012, 380:358-365.

17. Meckbach D, Keim U, Richter S, Leiter U, Eigentler TK, Bauer J, Pflugfelder A, Buttner P, Garbe C, Weide B: BRAF-V600 mutations have no prognostic impact in stage IV melanoma patients treated with monochemotherapy. PLoS One 2014, 9:e89218
18. Chen KG, Valencia JC, Gillet JP, Hearing VJ, Gottesman MM: Involvement of $A B C$ transporters in melanogenesis and the development of multidrug resistance of melanoma. Pigment Cell Melanoma Res 2009, 22:740-749.

19. Rockmann H, Schadendorf D: Drug resistance in human melanoma: mechanisms and therapeutic opportunities. Onkologie 2003, 26:581-587.

20. Sarasin A, Dessen P: DNA repair pathways and human metastatic malignant melanoma. Curr Mol Med 2010, 10:413-418.

21. Verbeek B, Southgate TD, Gilham DE, Margison GP: O6-Methylguanine-DNA methyltransferase inactivation and chemotherapy. Br Med Bull 2008, 85:17-33.

22. Bennett LL Jr, Smithers D, Rose LM, Adamson DJ, Shaddix SC, Thomas HJ: Metabolism and metabolic effects of 2-azahypoxanthine and 2azaadenosine. Biochem Pharmacol 1985, 34:1293-1304.

23. Szybalski W: Use of the HPRT gene and the HAT selection technique in DNA-mediated transformation of mammalian cells: first steps toward developing hybridoma techniques and gene therapy. Bioessays 1992, 14:495-500.

24. Talantov D, Mazumder A, Yu JX, Briggs T, Jiang Y, Backus J, Atkins D, Wang $Y$ : Novel genes associated with malignant melanoma but not benign melanocytic lesions. Clin Cancer Res 2005, 11:7234-7242.

25. Garnett MJ, Edelman EJ, Heidorn SJ, Greenman CD, Dastur A, Lau KW, Greninger P, Thompson IR, Luo X, Soares J, Liu Q, lorio F, Surdez D, Chen L, Milano RJ, Bignell GR, Tam AT, Davies H, Stevenson JA, Barthorpe S, Lutz SR, Kogera F, Lawrence K, McLaren-Douglas A, Mitropoulos X, Mironenko T, Thi H, Richardson L, Zhou W, Jewitt F, et al: Systematic identification of genomic markers of drug sensitivity in cancer cells. Nature 2012, 483:570-575.

26. Hoek KS, Schlegel NC, Brafford P, Sucker A, Ugurel S, Kumar R, Weber BL, Nathanson KL, Phillips DJ, Herlyn M, Schadendorf D, Dummer R: Metastatic potential of melanomas defined by specific gene expression profiles with no BRAF signature. Pigment Cell Res 2006, 19:290-302.

27. Hauser IA, Renders L, Radeke HH, Sterzel RB, Goppelt-Struebe M: Mycophenolate mofetil inhibits rat and human mesangial cell proliferation by guanosine depletion. Nephrol Dial Transplant 1999, 14:58-63.

28. Lev DC, Ruiz M, Mills L, McGary EC, Price JE, Bar-Eli M: Dacarbazine causes transcriptional up-regulation of interleukin 8 and vascular endothelial growth factor in melanoma cells: a possible escape mechanism from chemotherapy. Mol Cancer Ther 2003, 2:753-763.

29. Wouters J, Stas M, Gremeaux L, Govaere O, Van den Broeck A, Maes H, Agostinis P. Roskams T, van den Oord JJ, Vankelecom H: The human melanoma side population displays molecular and functional characteristics of enriched chemoresistance and tumorigenesis. PLoS One 2013, 8:e76550.

30. Metelmann HR, Von Hoff DD: In vitro activation of dacarbazine (DTIC) for a human tumor cloning system. Int J Cell Cloning 1983, 1:24-32.

31. Shibuya H, Kato Y, Saito M, Isobe T, Tsuboi R, Koga M, Toyota H, Mizuguchi $\mathrm{J}$ : Induction of apoptosis and/or necrosis following exposure to antitumour agents in a melanoma cell line, probably through modulation of Bcl-2 family proteins. Melanoma Res 2003, 13:457-464

32. Lev DC, Onn A, Melinkova VO, Miller C, Stone V, Ruiz M, McGary EC, Ananthaswamy HN, Price JE, Bar-Eli M: Exposure of melanoma cells to dacarbazine results in enhanced tumor growth and metastasis in vivo. J Clin Oncol 2004, 22:2092-2100.

33. Fiore D, Jackson AJ, Didolkar MS, Dandu VR: Simultaneous determination of dacarbazine, its photolytic degradation product, 2-azahypoxanthine, and the metabolite 5-aminoimidazole-4-carboxamide in plasma and urine by high-pressure liquid chromatography. Antimicrob Agents Chemother 1985, 27:977-979.

34. Parsons PG, Smellie SG, Morrison LE, Hayward IP: Properties of human melanoma cells resistant to 5-(3',3'-dimethyl-1-triazeno)imidazole-4-carboxamide and other methylating agents. Cancer Res 1982, 42:1454-1461.

35. Saunders PP, DeChang W, Chao LY: Mechanisms of 5-(3,3-dimethyl-1-triazeno)imidazole-4-carboxamide (Dacarbazine) cytotoxicity toward Chinese hamster ovary cells in vitro are dictated by incubation conditions. Chem Biol Interact 1986, 58(3):319-331.

36. Fellenberg J, Kunz P, Sahr H, Depeweg D: Overexpression of inosine 5'-monophosphate dehydrogenase type II mediates chemoresistance to human osteosarcoma cells. PLoS One 2010, 5:e12179.

37. Penuelas S, Noe V, Ciudad CJ: Modulation of IMPDH2, survivin, topoisomerase I and vimentin increases sensitivity to methotrexate in HT29 human colon cancer cells. Febs J 2005, 272:696-710.

38. Penuelas S, Noe V, Morales R, Ciudad CJ: Sensitization of human erythroleukemia K562 cells resistant to methotrexate by inhibiting IMPDH. Med Sci Monit 2005, 11:BR6-BR12. 
39. Aufderklamm S, Todenhofer T, Gakis G, Kruck S, Hennenlotter J, Stenzl A, Schwentner C: Thymidine kinase and cancer monitoring. Cancer Lett 2012, 316:6-10.

40. Verma S, Quirt IC, Eisenhauer EA, Iscoe NA, Young VJ, Bodurtha AJ, Davidson J: A phase II study of weekly edatrexate (10-EDAM) in metastatic melanoma: a national cancer institute of Canada clinical trials group study. Ann Oncol 1993, 4:254-255.

41. Leahy MF, Silver HK, Klimo P, Hall TC: Treatment of advanced malignant melanoma with high dose methotrexate and folinic acid rescue. Med Pediatr Oncol 1982, 10:151-156.

42. Chen KG, Valencia JC, Lai B, Zhang G, Paterson JK, Rouzaud F, Berens W, Wincovitch SM, Garfield SH, Leapman RD, Hearing VJ, Gottesman MM: Melanosomal sequestration of cytotoxic drugs contributes to the intractability of malignant melanomas. Proc Natl Acad Sci U S A 2006 103:9903-9907.

43. Saez-Ayala M, Fernandez-Perez MP, Montenegro MF, Sanchez-del-Campo L, Chazarra S, Pinero-Madrona A, Cabezas-Herrera J, Rodriguez-Lopez JN: Melanoma coordinates general and cell-specific mechanisms to promote methotrexate resistance. Exp Cell Res 2012, 318:1146-1159.

44. Sanchez-del-Campo L, Montenegro MF, Cabezas-Herrera J, Rodriguez-Lopez $\mathrm{JN}$ : The critical role of alpha-folate receptor in the resistance of melanoma to methotrexate. Pigment Cell Melanoma Res 2009, 22:588-600.

45. Saez-Ayala M, Montenegro MF, Sanchez-Del-Campo L, Fernandez-Perez MP, Chazarra S, Freter R, Middleton M, Pinero-Madrona A, Cabezas-Herrera J, Goding CR, Rodriguez-Lopez JN: Directed phenotype switching as an effective antimelanoma strategy. Cancer Cell 2013, 24:105-119.

46. Robien K, Boynton A, Ulrich CM: Pharmacogenetics of folate-related drug targets in cancer treatment. Pharmacogenomics 2005, 6:673-689.

47. Watson AJ, Margison GP: O (6)-alkylguanine-DNA alkyltransferase assay. Methods Mol Med 1999, 28:167-178

48. Gerson SL, Trey JE, Miller K, Berger NA: Comparison of O6-alkylguanine-DNA alkyltransferase activity based on cellular DNA content in human, rat and mouse tissues. Carcinogenesis 1986, 7:745-749.

49. Watson AJ, Margison GP: O6-alkylguanine-DNA alkyltransferase assay. Methods Mol Biol 2000, 152:49-61.

50. Elder RH, Margison GP, Rafferty JA: Differential inactivation of mammalian and Escherichia coli O6-alkylguanine-DNA alkyltransferases by O6-benzylguanine. Biochem J 1994, 298(Pt 1):231-235.

doi:10.1186/1476-4598-13-154

Cite this article as: Arozarena et al:: Differential chemosensitivity to antifolate drugs between RAS and BRAF melanoma cells. Molecular Cancer 2014 13:154.

\section{Submit your next manuscript to BioMed Central and take full advantage of:}

- Convenient online submission

- Thorough peer review

- No space constraints or color figure charges

- Immediate publication on acceptance

- Inclusion in PubMed, CAS, Scopus and Google Scholar

- Research which is freely available for redistribution 